\title{
Iris Segmentation Based on Black Hole Algorithm for Biometric System
}

\author{
Tara Othman Qadir, N.S.A.M Taujuddin
}

\begin{abstract}
Current iris recognition schemes such as IntegroDifferential method, Hough Transform, Watershed Transform Circle Fitting, and Circular Hough Transformation (CHT) are used to find circular parameters between pupil and iris. Segmentation process of an eye image using the circular parameters toextracts the iris region still can be further improved. In this paper, we introduced an optimization method of circular parameters detection for iris segmentation based on Black Hole Algorithm (BHA). The proposed segmentation algorithm utilizes a computational model of the pixels' value to detect the iris boundary. The BHA searches for center radius of both pupil and iris. The system tests the CASIA Iris Interval V3 database by on MATLAB. The segmented images show an accuracy of $98.3 \%$. In short, the segmentation-based on $B H A$ is efficient to identify the iris for any future access control applications.
\end{abstract}

Keywords: Black Hole Algorithm, Biometric, Iris recognition, Image processing, Segmentation.

\section{INTRODUCTION}

$\mathrm{R}$ various security applications. The growing market demand for an automated access control systems based on biometric recognition platform motivates this research [1]. Many identification features have been explored for human recognition systems, such as iris, fingerprint, face, mouth, and ear [2]. However, the personal identification systems based on iris are the most stable and reliable recognition recognition system was described by Daugman in 1993 [4]. The iris recognition systems include several stages, such as edge localization, segmentation, normalization, features extraction, and matching. High segmentation accuracy can significantly improve the recognition system outcomes. The features of iris texture are different between any two persons and the between the left and right eye of the same person too. Figure. 1 illustrates a sample of the tested database of iris images [5]. It can be noted that the small black circle is the pupil of the eye, and the white area is the sclera.

Revised Manuscript Received on July 22, 2019

* Correspondence Author

Tara Othman Qadir*, her postgraduate at this College of Science and was awarded M.SC. degree in Computer Science , University of Sulaimani City Sulaimani, Lecturer in Salahaddin University Erbil in Collge of Engineering department Software Engineering ,in city Erbil in Country.Iraq , Email:alsaraftara@gmail.com

N. S. A. M, Taujuddin, Faculty of Electrical and Electronic Engineering, Universiti Tun Hussein Onn Malaysia, 86400 Parit Raja, Batu Pahat, Johor, Malaysia. Email: shahidah@uthm.edu.my

(C) The Authors. Published by Blue Eyes Intelligence Engineering and Sciences Publication (BEIESP). This is an open access article under the CC BY-NC-ND license (http://creativecommons.org/licenses/by-nc-nd/4.0/) biometric [3]. The first successful implementation of an iris

However, the region between the pupil and sclera is the iris of the eye. The identify of both inner and outer boundaries of the irisregion accurately is the main challenging to increase the identification accuracy. However, it is common to test a partial iris image. Whereas both the eyelid and eyelash are occluded the region of interest (ROI) for an iris. The conventional localization methods fail to localize the ROI outer boundary (the large circle). Moreover, the inner boundaries' radius, center, and shape of iris ROI are always changed due to the light intensity on the eye pupil. However, the parameters of both inner and outer circular need to obtain individually due to incompatible boundaries and centres [6]. Therefore, the accurate segmentation of the partial iris boundaries is a significant issue of the biometric iris recognition systems.

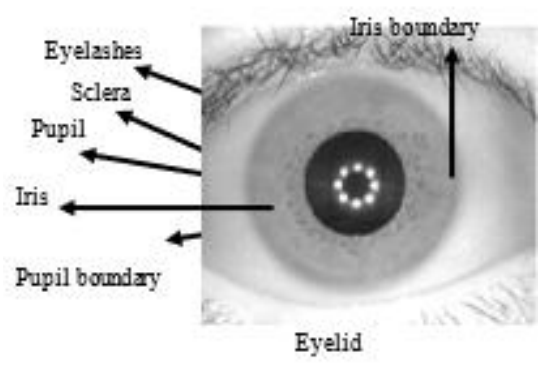

Fig. 1. Sample of eye image sections [5]

An accurate segmentation method is necessary for high accuracy iris system. The iris segmentation may cost half of the total time and increase the computation complexity of the system. Therefore, several studies have addressed the iris segmentation issue in the literature. Daugman proposed iris recognition system based on an Integro-differential method to obtain the boundary of the iris [4]. After that, Wildes introduced the Hough transform method to find the iris boundaries [7][8][9]. These two conventional methods need to search for all parameter space probability of the iris boundaries' parameters. In 2016, the authors located the outer circle using the Watershed transform and circle fitting, while the inner circle is detected by using the Canny edge and fitting circle method [10]. In 2017, Jan proposed an iris localization method for non-ideal iris data [11]. The visible wavelength illumination used to locate the iris boundaries. The computation of both image intensity and IntegroDifferential Operator (IDO) were used for edge detection and identify the inner and outer circles. In addition, Memar introduced an adaptive intensity threshold method to obtain the pupil and iris edge [12]. The inner and outer circles boundaries werelocalized by using circular Hough transformation (CHT). In 2018, Sardar [13] proposed iris segmentation-based rough entropy and hole filling

\section{Published By:}

Blue Eyes Intelligence Engineering and Sciences Publication

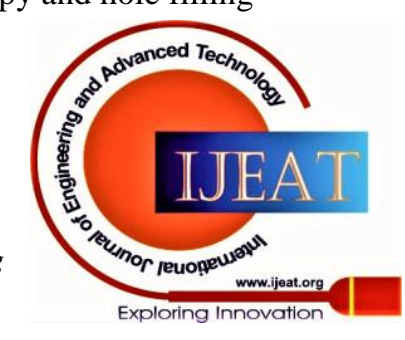




\section{Iris Segmentation Based on Black Hole Algorithm for Biometric System}

binarization for the inner circle. However, the outer circle is calculated approximately from the sclera edge. This method achieves an accuracy of $97.12 \%$ using CASIA-V3-Interval database. However, using the binarization scheme is not enough for next step normalization process. The normalization using Daugman-Rubbersheet needs the parameters of both inner and outer circles, as discussed in [14].

The Black Hole Algorithm (BHA) proposed in [15] for data clustering. The BHA has a simple structure and easy to implement. It has a fast searching process for optimal parameters of an objective function. In this article, the iris circular parameters inner and outer circles are obtained by using the BHA. This paper includes several sections as follows: Section II describes the mechanism of BHA. Section III explores the methodology of the iris segmentation. In Section IV, the results and the discussion of the proposed model are addressed. Finally, Section V concludes this paper.

\section{BLACK HOLE ALGORITHM}

Last two century, Michell and Laplace [16] identify the principle of the Black Hole $(\mathrm{BH})$ in the space. Theoretically, the light of the stars cannot pass, and it attracted to the $\mathrm{BH}$. The massive gravity is observed due to squeezing the large mass matter into a small space. The phenomenon described that the $\mathrm{BH}$ is swallowed anything moving close to the $\mathrm{BH}$ (inside the $\mathrm{BH}$ boundary), then it disappears. The BH boundary is formed in a spherical shape that is called the event horizon. The radius of the event horizon is calculated by $(1)$, as follows $[16,17]$

$$
R=\frac{2 G M}{c^{2}}
$$

where $\mathrm{G}, \mathrm{M}$, and c are the gravitational constant, mass, and the speed of light.

The BHA is a population-based method that the candidates are generated and distributed randomly in the search space [15]. Then, the candidates move toward the best candidate that has the lowest cost fitness from the previous iteration. Equation (2) illustrates the movement of the stars toward the black hole. The Euclidean distance between the $\mathrm{BH}$ and each star is calculated. Any star is founded in the $\mathrm{BH}$ regain (event horizon) is terminated from the optimization. In the next iteration, new stars are randomly generated within the boundary of the $\mathrm{BH}$ region. Then, the fitness values of all the new candidates are evaluated again. The candidate with the lowest fitness becomes a new $\mathrm{BH}$. In the optimization, the event horizon radius is called the Schwarzschild radius. In the real space, the Schwarzschild radius is computed by (1) while in BHA is computed by (3) [15]:

$$
X_{i}(t+1)=X_{i}(t)+\operatorname{rand} \times\left(X_{B H}-X_{i}(t)\right) i=1,2, \ldots, N
$$

$$
R=\frac{f_{B H}}{\sum_{i=1}^{N} f_{i}}
$$

where $\mathrm{Xi}(\mathrm{t})$ and $\mathrm{Xi}(\mathrm{t}+1)$ signify the locations of the ith star at iterations $t$ and $t+1$, respectively. Moreover, rand indicates uniform distribution with a range from 0 to 1 . $N$ denotes the number of stars. XBH points the location of the black hole in the exploration space. $\mathrm{R}$ is the radius of the event horizon, fi denotes the fitness value of the ith star, and $\mathrm{fBH}$ indicates the fitness value of the black hole.

\section{IRIS SEGMENTATION BASED BLACK HOLE ALGORITHM}

\section{A. Submission of the paper}

The framework of the proposed model is illustrated in Fig. 2. The segmentation model based BHA optimization is demonstrated with several steps, such as choosing the database, pre-processing, circular boundary, and BHA optimization of circular parameters.

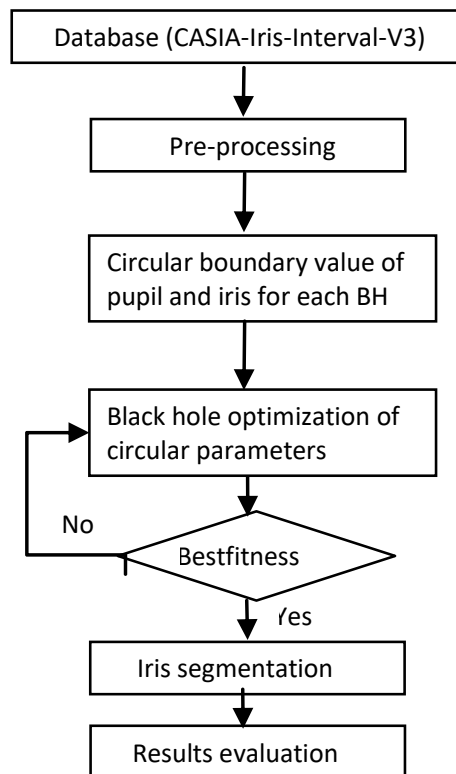

Fig. 2. The framework of iris segmentation

\section{B. Database}

The CASIA-Iris-V3 interval database is utilised to test the proposed iris segmentation model [5]. All iris images are 8 bit grey-level JPEG files, collected under near infrared illumination. The total number of images are 2655 , taken fromr 249 subjects with the image resolution of $320 \times 280$.

\section{Pre-processing}

The following processes are implemented before testing an image. Firstly, the tested image is loaded into MATLAB workspace. Secondly, the name of the tested image is recorded. Fig. 3 displays one of the tested images (S1081R08.jpg) from the CASIA database. Thirdly, the image size is checked. If the image has three layers, it will convert to grey-scale. After that, the image matrix with its pixels' values is imported into the MATLAB workspace. Now, the imparted image matrix is ready to obtain the boundary of the iris image.

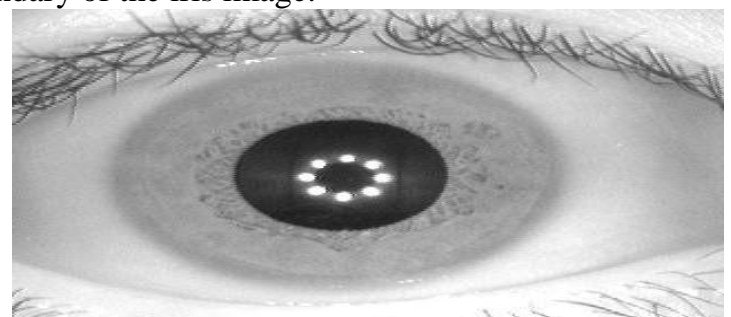

Fig. 3. The image S1081R08.jpg from CASIA iris database [5]

Published By:

Blue Eyes Intelligence Engineering and Sciences Publication

(C) Copyright: All rights reserved.

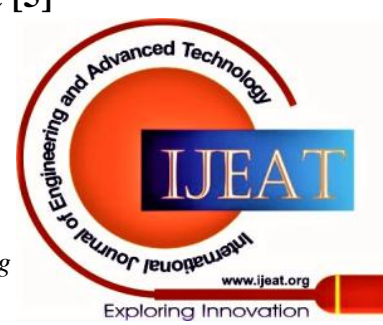




\section{Circular Boundary Detection}

The boundary detection of the iris image based on pixels' values is considered as the first issue in this paper. For example, the one-dimensional illustrations of the iris image vector. Here, we may intuitively say that there should be a boundary pixel between the 5th and 6th pixels.

\section{\begin{tabular}{ll|l|l|l|l|l|l|l|}
53 & 58 & 55 & 62 & 78 & 123 & 151 & 145 & 141 \\
\hline
\end{tabular}}

If the intensity difference were higher between the 5th and the 6th pixels, and if the intensity differences between the adjacent neighbouring pixels (1st to 4th and 6th to 9th) were smaller, it would not be as easy to say that there should be a boundary in the corresponding region. Moreover, one could argue that this case is one in which there are several edges. The 5th pixel is considered as a boundary that can be computed by using Equation (4).

$$
f(x)=\frac{\sum_{i=x+1}^{i=x+n} I(i)-\sum_{i=x-1}^{i=x-n} I(i)}{n}
$$

where $f(x)$ is the boundary value, $I(i)$ is the value of a particular pixel, and $n=4$ is the 4 th order detection. The histogram analysis is implemented for the tested image by using MATLAB to analyse the pixels population. Fig. 4 display three population of three important region in the image (pupil, iris, and sclera). Hence, a specific threshold on how large the intensity change between two neighbouring pixels must be calculated. Therefore, two thresholds values are needed to find the boundary of both pupil and iris. These thresholds can be obtained by using multi-level Otsu's method [18] in MATLAB, where the tested images are analyzed using Otsu method. The outcomes of the multilevel thresholds are obtained as follows: the pixels value of pupil region is (20 to 61), iris region (124 to 165), and the sclera region (185 to 219). Therefore, according to Equation (4) the minimum value $f(x)=63$ of the pupil boundary's pixels, and the minimum value $f(x)=20$ of the iris boundary's pixels. According to that, the $\mathrm{f}(\mathrm{x})$ of BHA is set with limit optimization between 20 and 70 in MATLAB. During the optimization, the $f(x)=(20$ to 70$)$ of any pixel is considered as boundary pixel.

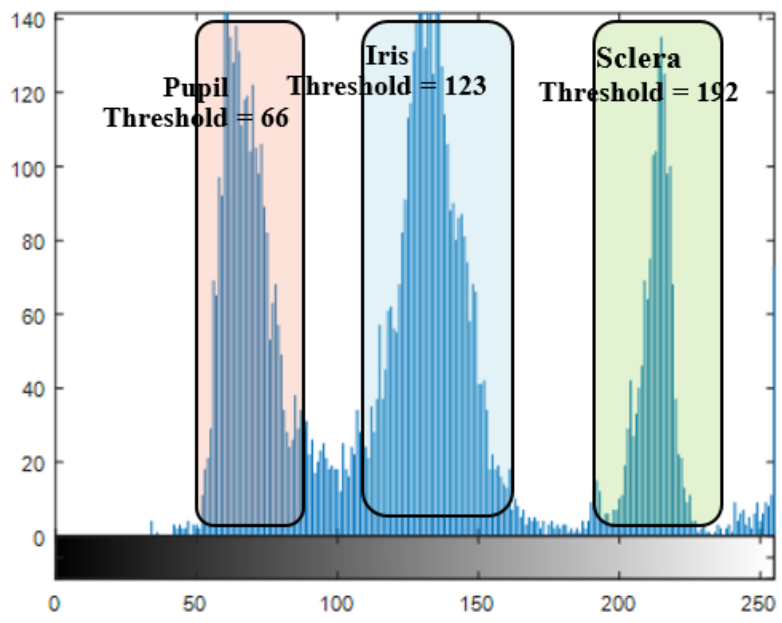

Fig. 4. Histogram pixels' values of the pupil, iris, and sclera.

The process of detection of the boundary pixels is implemented four times for different angles $0^{\circ}$ and $180^{\circ}, 45^{\circ}$ and $225^{\circ}, 90^{\circ}$ and $270^{\circ}, 135^{\circ}$ and $315^{\circ}$. That can increase the accuracy of the calculation for different directions of boundaries, as displayed in Fig 5.

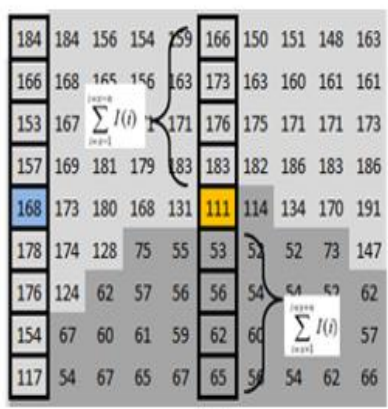

(a)

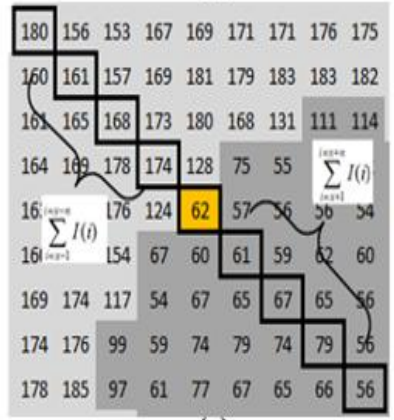

(c)

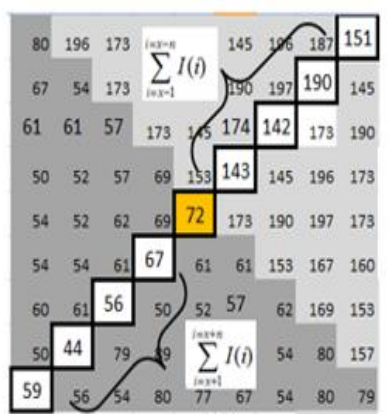

(b)

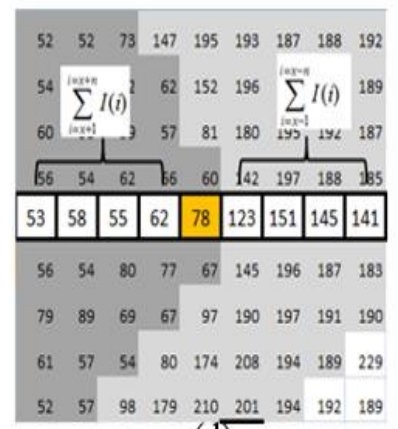

(d)

Fig.5. Boundary pixels recognition at different angles.

\section{E. Example of pixel value}

For the vertical axis Fig.5 (a) the yellow pixel is given a high value of $\mathrm{f}(\mathrm{x})=116$ according to equation (4).

$f(x)=\frac{(166+173+176+183)-(53+56+62+65)}{4}$

$$
=116
$$

Therefore, it is considered as pixel on the boundary of the pupil. On the other hand, the blue cell in Fig.5 (a) results a low value according to the calculation of equation (4), where the value of $\mathrm{f}(\mathrm{x})=8.8$.

$f(x)$

$$
=\frac{(184+166+153+157)-(178+176+154+117)}{4}
$$

So that, it's recognised as non boundary pixel.

\section{F. Black hole optimization for pupil and iris boundary}

The BHA search method is implemented in this study for iris segmentation. The BHA can reach the optimum parameters in a short time and less computational process. Firstly, the circle equation (5) is set as the objective function of the optimization problem. The algorithm searches for the three main parameters of the pupil circle Rp, Xp, and Yp where $\mathrm{Rp}$ is the radius of the pupil circle in the pixel unit. Moreover, Xp and Yp represent the location of the circle centre. Secondly, the computing of boundary pixel value equation (4) is utilized to analyses the results and calculated the fitness function. Thirdly, the BHA generates randomly 100 stars (100 circles) of each iteration, as shown in Fig. 6. Each candidate (star is a circle) gets random values of Rp, $\mathrm{Xp}$, and Yp. The size of the image is considered during the optimization to make all the suggested 100 circles are inside the image.

\section{Published By:}

Blue Eyes Intelligence Engineering and Sciences Publication

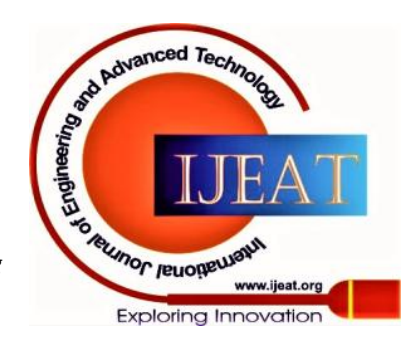




\section{Iris Segmentation Based on Black Hole Algorithm for Biometric System}

The star parameters demonstrate a circle with 360 step size (one pixel every one degree) by using (5). Fourthly, this circle is plotted in a black mask zeros image (Istar) that has the same size as the input image $(320 \times 280)$. However, same circle is generated on the original image without touching the image boarders. The pixels that located at the boundary of the circle are computed by using equation (4). The maximum value of the equation (4) can be achieved when the pixel is found at the boundary between the pupil and the iris or between the iris and the sclera. Fifth, the computed value of the pixel using equation (4) higher than the thresholds is counted and recoded. This calculation is implemented for different directions around this pixel. Same process is implemented for the others pixels, which are located at the boundary of this circle. The number of recoded pixels (circle boundary is true) for this star is computed. Sixth, the result of this candidate shows only the recoded pixels. The fitness value of the first star (first circle) for not recoded pixels is calculated by using equation (6).

$$
\begin{aligned}
& x=r \times \cos (\theta)+c x, \text { and } y=r \times \sin (\theta)+c y \text {. } \\
& f_{\text {star }}=1-\frac{P_{\text {recoded }}}{P_{\text {star }}}
\end{aligned}
$$

where $r=R p, \theta$ is the angle, $c x=X p, c y=Y p$, fstar is the fitness value of the star, Precoded is the total number of recoded pixels, and Pstar $=360$ is the number of star's pixels. For example, Fig. 6(a) illustrates the star number 7 that is generated by the first iteration of BHA. The 7th star in Fig. 6(a) is represented as a yellow circle on the tested iris image. The boundary of the pupil is intersect the yellow circle boundary at two pointes only. According to that, the calculation of Equation (6) gives a high fitness value f7th star $=1-(47 / 360)=0.87$. In addition, the calculation of the star number 31 gives a fitness value of f31th star $=1$ $(61 / 360)=0.83$, as shown in Fig. 6(b). The calculation of the star number 57 gives a fitness value of f57th star $=1$ $(143 / 360)=0.60$, as shown in Fig. 6(c). Moreover, the calculation of the star number 73 gives a fitness value of f73th star $=1-(257 / 360)=0.28$, as shown in Fig. 6(d). According to the proposed framework in Fig 2, the generated circle of the star number 73 observes the lowest cost fitness. Therefore, the star no 73 is considered as a black hole of the pupil for the second iteration. In the second iteration, a new 100 stars are also generated by using Equation (2) BHA. However, these new stars have a circular parameters close to the star number 73 of the first iteration. The stars parameters have conditions of horizon radius of BHA according to Equation (3). Therefore, all the new suggested circles will be allocated close to the pupil boundary. As a results, the best circle that is represented the pupil boundary is detected in short time around 2 Sec.

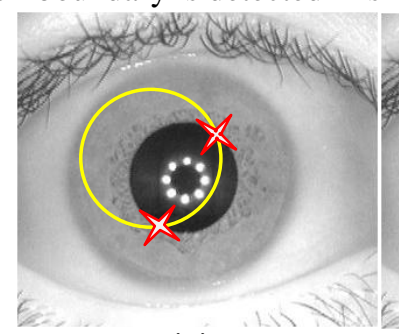

(a)

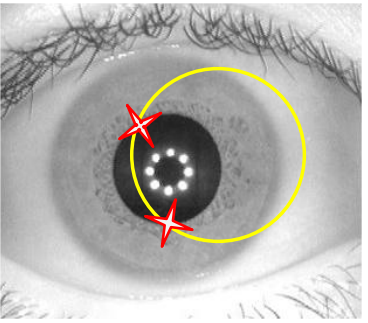

(b)

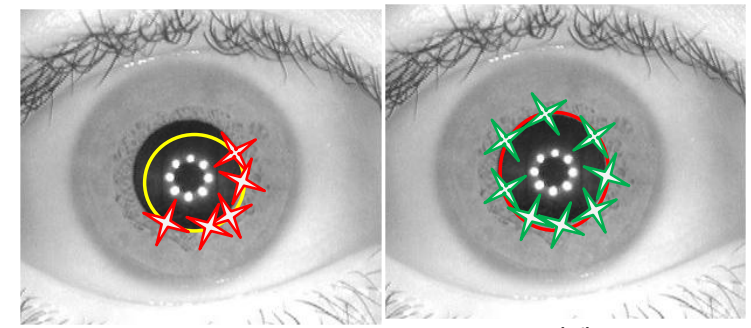

(c)

(d)

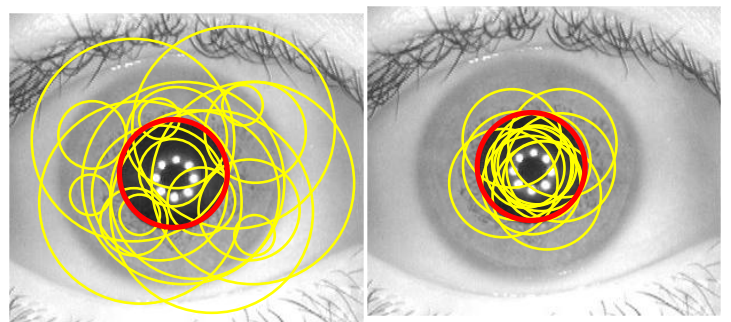

(e)

$(f)$

Fig. 6. The BHA operation, (a) star number 7, (b) star number 31, (c) star number 57, (d) star number 73 which is the BH of the first iteration, (e) first iteration the BHA generates randomly 100 stars (100 circles), and (f) second iteration generates $\mathbf{1 0 0}$ circles close to the pupil.

This process is implemented for all the other 99 stars and the fitness values of the all stars. Seventh, the BHA evaluates the fitness values for all the stars to update the location and the black hole candidate for the next iteration. Eighth, the star with fitness value less than the fitness of the current black hole becomes the new black hole in the next iteration. In the next iteration, the new random 100 stares move toward the new black hole. The BHA generates another 100 circles with parameters that are close to the parameters of the new $\mathrm{BH}$ (has best fitness from last iteration). Then, the eight steps that are implemented in the first iteration are processed again. The best fitness star of the second iteration becomes the $\mathrm{BH}$ for the third iteration, and so on. In the third iteration, the location of the $\mathrm{BH}$ is also updated, and the stars become closer to the perfect boundary of iris. Finally, this algorithm can find the optimum parameters shortly without computing all the probabilitiesof Rp, Xp, and Yp. Fig. 7 displays the identified circle of the pupil boundary.

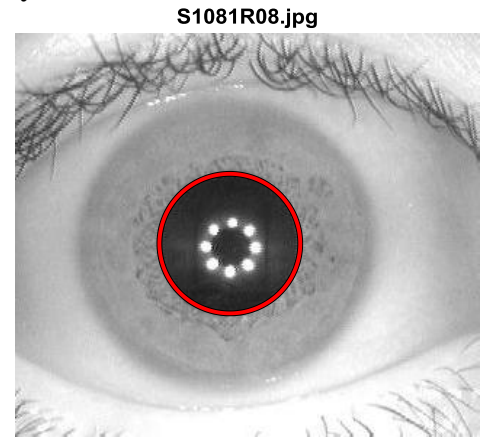

Fig. 7. Pupil boundary identification

\section{G. Black hole optimization for iris boundary}

For iris boundary, same process that illustrated in the last section is implemented.

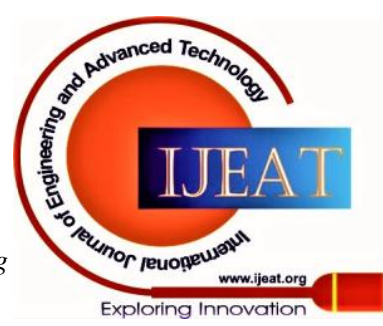


The BHA can suggested a random circle parameters for each star. The best fitness star in the first iteration becomes the black hole for the next iteration. One by one, the fitness value of the objective function is improved. Once the stop criteria are achieved, the optimum circle is give that presented the iris boundary, as depicted in Figure. 8. The BHA has obtained the center and radius of the iris boundary accurately, as displayed in Figure. 8.

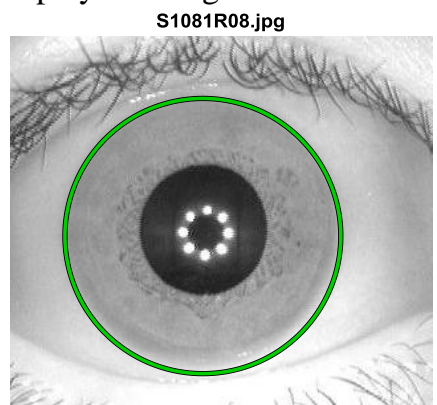

Figure. 8. Iris boundary identification

\section{H. Iris mask}

A logical array mask is obtained for two reasons. Firstly, it is used to extract only the iris segmented region. Secondly, it is used to evaluate the accuracy of the proposed segmented system. The mask image (Imask) is generated by creating a zeros array $320 \times 280$. After that, 1 logical values are set on the region of the iris by ploting a filled circule on the Imask. Then, 0 logical values are set on the region of the pupil by ploting a filled circule on the Imask. The proposed mask of the segmented iris image (Imask) is multiplied with the input image (Iinput) by using element-wise multiplication. Where only the segmented iris region is displayed (Isegmented), as depicted inFig. 9.

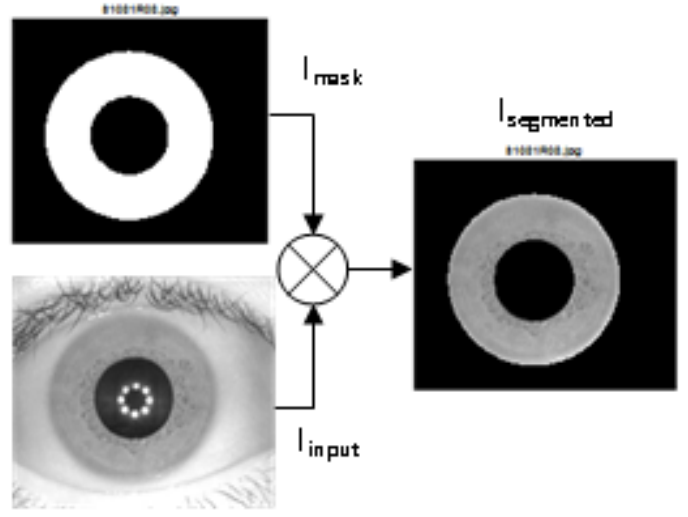
Fig. 9. Masking the input image process and output
segmented image

Based on the discussed framework the main steps of BHA segmentation are summarized as below:

Algorithm: Pseudocode of BHA segmentation

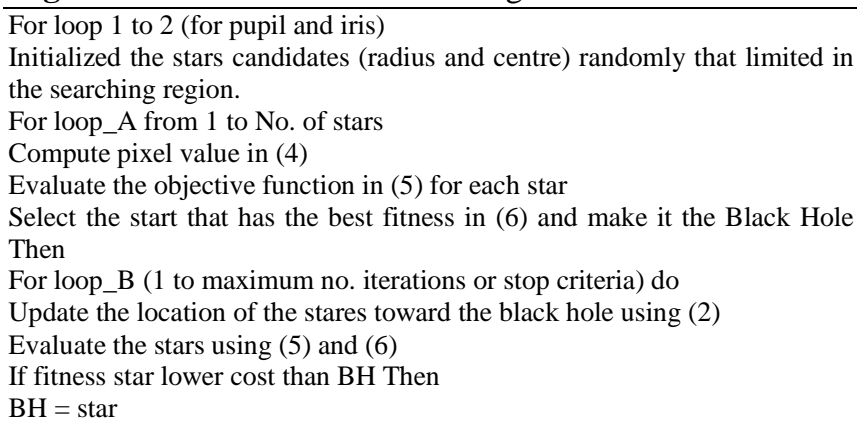

End If

Calculate R from (3)

ace the star with a new star

End If

If stop criteria true

Stop loop_B

End loop_B

A

(centre and radius) and fitness values for pupil and iris

logical mask image (Imask) Then

Isegmented $=$ Iinput $\otimes$ Imask

mask error $=($ Imask XOR IGT)

Acc $=$ Number of correct segmented images / Total number of images $\times$ $100 \%$

End

\section{EXPERIMENTAL RESULTS}

The available iris database is used in this experiment. CASIA iris interval V3 database is selected to test the proposed segmentation method. This database is selected because it includes 2639 images, where most of the images in CASIA have non-ideal iris (partial occlusion).

The proposed segmentation method is implemented using MATLAB 9.6 (R2019b), a computer with $2.1 \mathrm{GHz}$ processor and 4 GB RAM. Figure 10 depicts some of the images that segmented using the BHA. The result demonstrates that both the pupil and the iris boundaries are detected with high accuracy. Although Figure 10 shows images with different occlusion and radius of pupil or iris, the proposed algorithm is successfully segmented the iris region.

In addition, the average accuracy of the proposed algorithm is evaluated with the model in [13]. The Ground Troth images (IGT) of CASIA iris interval V3 in [19] is utilized to test the accuracy of the extracted mask (Imask) individually. Whereas themismatch localized pixels (Imismatch) are obtained byusing Imask XOR IGT operation. The performance matrix is calculated by using confusionmat function of Imask and IGT. The accuracy of the segmented image represents the percentage of correct predictions pixels, as presented in (7), where TN, TP, FN, FP denotes true negative, true positive, false negative and false positive, respectively. It is calculated that the accuracy is $98.54 \%$ of segmented image S1081R08 using BHA, as displayed in Fig. 11 (a).

Accuracy $=(T P+T N) /(T P+T N+F N+F P)$. (7)

The Imismatch images are used to find the average accuracy of all the images. In case segmentation of an image has accuracy less than $90 \%$ using (7) is considered as incorrect segmented image (FN). However, any image segmented with accuracy higher than 90\% using (7) is counted as correct segmentation (TP). In this experiment, many images of different subjects are selected randomly to test the average accuracy of the BHA. Fig. 11 (b) illustrates the confusion matrix of the tested images. The average accuracy of all tested images is calculated in (8).

Average accuracy $=\frac{\text { Number of correct segmented images }}{\text { Total number of images }} \times 100 \%$

(8)

(C) Copyright: All rights reserved.

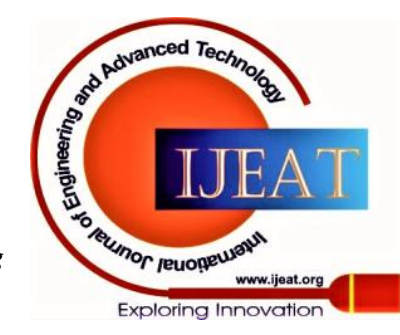




\section{Iris Segmentation Based on Black Hole Algorithm for Biometric System}

The proposed BHA segmentation achieves an average accuracy of $98.3 \%$. The proposed $\mathrm{BH}$ algorithm shows the average accuracy of segmentation higher than the accuracy in [13]. However, the possessing time $=2.9 \mathrm{sec}$ of $\mathrm{BHA}$ is a bit longer. It can argue that for several rezones, such as a high number of iteration loop without stop criteria, the BHA obtained the fitness of each star while the rough entropy in [13] made binarization image, and slow processing of our computer.
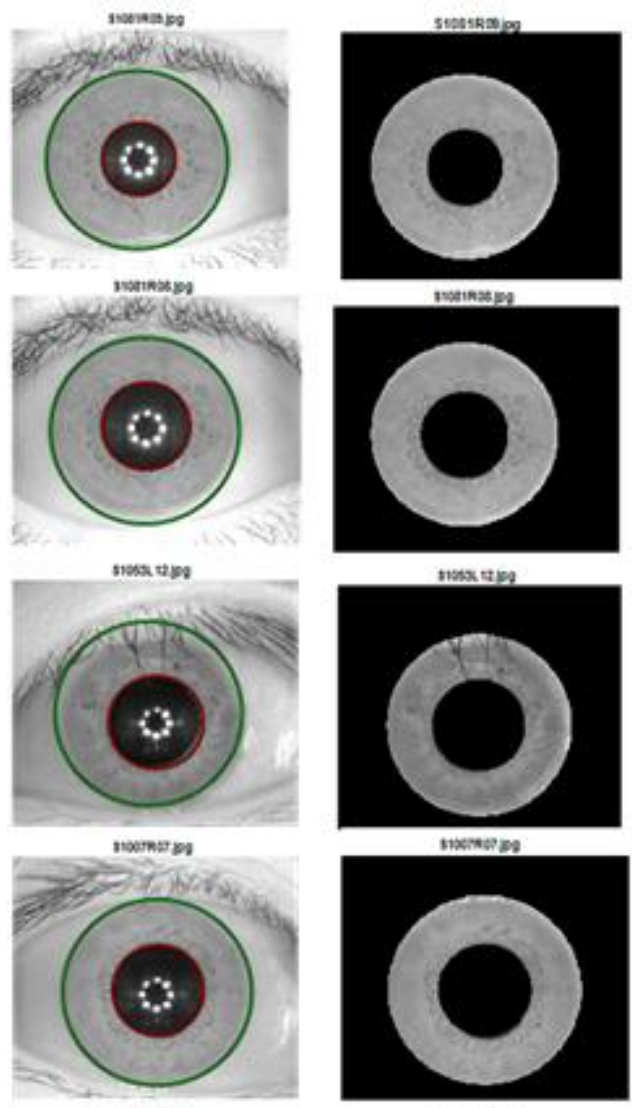

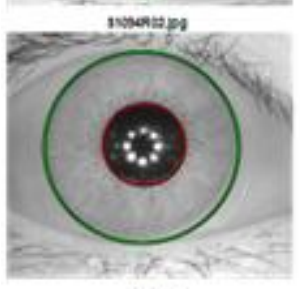

(a)

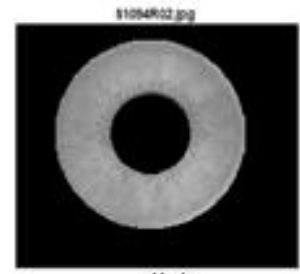

(b)
Fig. 10. Sample of CASIA image (a) boundaries detection (b) iris segmented

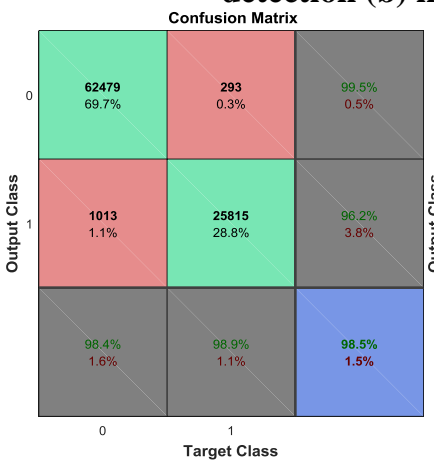

(a)

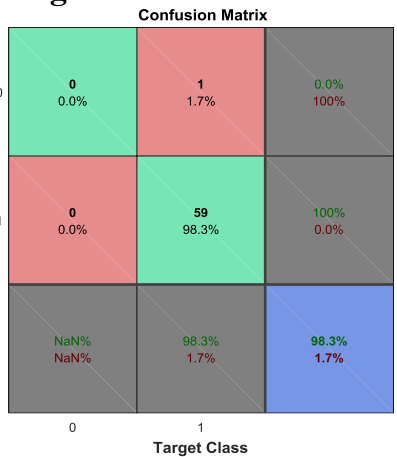

(b)
Fig. 11. The confusion matrix of (a) of image S1081R08 (b) of the all tested images

The CASIA iris interval V3 database has segmented accurately by using Black Hole algorithm. The Black Hole optimization is developed to segment the pupil and the iris boundaries. In the optimization, the full boundary of the pupil was considered. High accuracy of $98.3 \%$ segmentation has achieved by using pixels value and black hole algorithm. Whereas the optimization time of 2.9 second was acceptable comparing with the state-of-the-art method (Need to list down methods that you compare with BHA). The proposed system segmented the circular boundaries only while removing the noise of both the eyelid and eyelash considered in our future work. The proposed algorithm can be used to improve the efficiency of any iris biometric security system.

\section{REFERENCES}

1. R. Tobji, W. Di, N. Ayoub, and S. Haouassi, "Efficient Iris Pattern Recognition Method by using Adaptive Hamming Distance and 1D Log-Gabor Filter," INTERNATIONAL JOURNAL OF ADVANCED COMPUTER SCIENCE AND APPLICATIONS, vol. 9, no. 11, pp. 662-669, 2018.

2. M. Haghighat, M. Abdel-Mottaleb, and W. Alhalabi, "Discriminant correlation analysis: Real-time feature level fusion for multimoda biometric recognition," IEEE Transactions on Information Forensics and Security, vol. 11, no. 9, pp. 1984-1996, 2016.

3. N. Ahmadi and G. Akbarizadeh, "Hybrid robust iris recognition approach using iris image pre-processing, two-dimensional gabor features and multi-layer perceptron neural network/PSO," IET Biometrics, vol. 7, no. 2, pp. 153-162, 2017.

4. J. G. Daugman, "High confidence visual recognition of persons by test of statistical independence," IEEE transactions on pattern analysis and machine intelligence, vol. 15, no. 11, pp. 1148-1161, 1993.

5. T. Tan, "Note on CASIA-IrisV3," Center for Biometrics and Security Research, National Laboratory of Pattern Recognition Institute of Automation, Chinese Academy of Sciences, 2011.

6. K. Hajari and K. Bhoyar, "A review of issues and challenges in designing iris recognition systems for noisy imaging environment," in International Conference on Pervasive Computing (ICPC), Pune, India, 8-10 January 2015, pp. 1-6.

7. H. G. Daway, H. H. Kareem, and A. R. Hashim, "Pupil Detection Based on Color Difference and Circular Hough Transform," International Journal of Electrical and Computer Engineering, vol. 8 no. 5, pp. 3278-3284, 2018.

8. L. Zhong, K. Meng, Y. Wang, Z. Dai, and S. Li, "Iris Location Algorithm Based on the CANNY Operator and Gradient Hough Transform," in IOP Conference Series: Materials Science and Engineering, 2017, pp. 1-7.

9. R. P. Wildes, "Iris recognition: an emerging biometric technology," Proceedings of the IEEE, vol. 85, no. 9, pp. 1348-1363, 1997.

10. M. Frucci, M. Nappi, D. Riccio, and G. S. di Baja, "WIRE: Watershed based iris recognition," Pattern Recognition, vol. 52, pp. 148-159, 2016.

11. F. Jan, "Non-circular iris contours localization in the visible wavelength eye images," Computers \& Electrical Engineering, vol. 62, no. 2, pp. 166-177, 2017.

12. S. Memar Zadeh and A. Harimi, "Iris localization by means of adaptive thresholding and Circular Hough Transform," Journal of AI and Data Mining, vol. 5, no. 1, pp. 21-28, 2017.

13. M. Sardar, S. Mitra, and B. U. Shankar, "Iris localization using rough entropy and CSA: A soft computing approach," Applied Soft Computing, vol. 67, pp. 61-69, 2018.

14. H. K. Rana, M. S. Azam, M. R. Akhtar, J. M. Quinn, and M. A. Moni, "A fast iris recognition system through optimum feature extraction," PeerJ Computer Science, vol. 5, no. e184, pp. 1-5, 2019.

15. A. Hatamlou, "Black hole: A new heuristic optimization approach for data clustering," Information sciences, vol. 222, pp. 175-184, 2013.

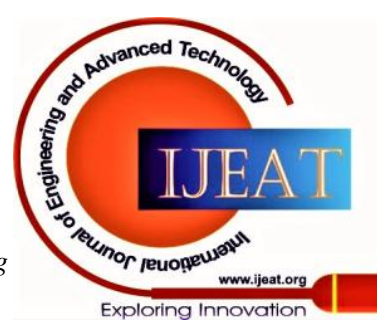


16. R. Giacconi, Black hole research past and future, in: black holes in binaries and galactic nuclei: diagnostics, demography and formation, 1st ed. Garching, Germany: Springer, 2001.

17. C. A. Pickover, Black holes: A traveler's guide. United States of America: John Wiley \& Sons, 1998.

18. S. C. Satapathy, N. S. M. Raja, V. Rajinikanth, A. S. Ashour, and N. Dey, "Multi-level image thresholding using Otsu and chaotic bat algorithm," Neural Computing and Applications, vol. 29, no. 12, pp. 1285-1307, 2018.

19. H. Hofbauer, F. Alonso-Fernandez, P. Wild, J. Bigun, and A. Uhl, "A ground truth for iris segmentation," in 22nd international conference on pattern recognition, Stockholm, Sweden, 24-28 August 2014, pp. $527-532$

\section{AUTHORS PROFILE}

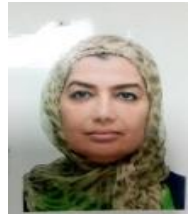

Tara Othman Qadir, her PHD from Faculty of Electrical and Electronic Engineering, University Tun Hussein Onn Malaysia (UTHM),M.SC. degree in Computer Science, University of Sulaimani City SulaimaniBachelor Degree from UniversityBagdad, Lecturer in Salahaddin University in Erbil in College of Engineering department Software Engineering ,in city Erbil in Country. Iraq , Email:alsaraftara@gmail.com

Research Interest Pattern recognition, computervision, machine learning

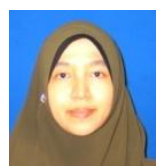

Nik Shahidah Afifi Md Taujuddin obtained her PhD from Universiti Tun Hussein Onn Malaysia, while Master and Bachelor Degree from Universiti Teknologi Malaysia in Electrical and Electronic Engineering. She also used to be a visiting researcher at Nagaoka University of Technology, Japan. Recently, she taught at Universiti Tun Hussein Onn Malaysia.

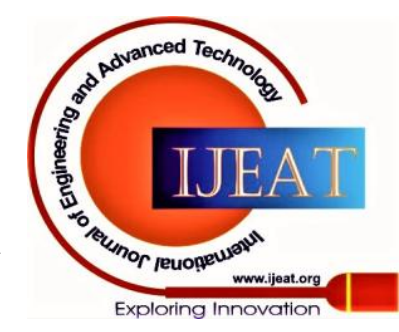

